\title{
Colección de Mamíferos, Museo de Historia Natural de la Universidad Distrital Francisco José de Caldas (MHNUD-M)
}

\author{
Abelardo Rodríguez-Bolaños ${ }^{1 *(i D}$ \\ 1 Facultad de Ciencias y Educación, Universidad Distrital Francisco José de Caldas, Museo de Historia Natural, Laboratorio \\ Biodiversidad de Alta Montaña. Carrera 3 No. 26A-40. Edificio de laboratorios de Biología-Primer Nivel. Bogotá, Colombia. \\ *Correspondencia: arodriguezb@udistrital.edu.co
}

\section{Resumen}

La Colección de Mamíferos del Museo de Historia Natural de la Universidad Distrital Francisco José de Caldas (MHNUD-M) alberga 2.030 especímenes de mamíferos asignados a 13 órdenes, 36 familias, 117 géneros y 223 especies. Esta colección se fundó en 1999 con la colecta de un ejemplar de Nephelomys childi del sector de Fruticas en el noreste del páramo de Sumapaz (Cundinamarca, Colombia). La colección alberga especímenes provenientes de 17 departamentos con un énfasis en los mamíferos de la alta montaña colombiana. Es una de las colecciones más jóvenes del país y sus ejemplares han sido citados en al menos 18 publicaciones científicas.

Palabras clave: Alta montaña, Chiroptera, Especímenes, micromamíferos terrestres.

\section{Abstract}

The Mammal Collection of the Natural History Museum of the Universidad Distrital Francisco José de Caldas (MHNUD-M) holds 2.030 specimens of mammals belonging to 13 orders, 36 families, 117 genera, and 223 species. This collection was founded in 1999 with the collect of Nephelomys childi specimen from the Fruticas sector in the northeast part of the Páramo de Sumapaz. Today the collection houses specimens from 17 departments with a strong emphasis on mammals of the Colombian high mountains. It is one of the youngest collections in the country and specimens have been cited in at least 18 scientific publications.

Key words: Chiroptera, high mountain, specimens, terrestrial micromammals.

La Colección de Mamíferos del Museo de Historia Natural de la Universidad Distrital Francisco José de Caldas (MHNUD-M) (Figura 1 y 2) se ubica en la sede La Macarena en la ciudad de Bogotá. Fue fundada en 1999 y en la actualidad preserva 2.030 especímenes de mamíferos (registro al 30 de marzo de 2020) catalogados y 34 especímenes en proceso de ingreso al catálogo de la colección. La colección cuenta con una representación taxonómica de 13 órdenes, 36 familias, 117 géneros (uno exótico) y 223 especies (Tabla 1). En total, 1.743 ejemplares se encuentran catalogados a especie (287 por identificar a especie), 2.028 hasta género y dos ejemplares sólo han sido identificados a nivel de familia. 
Los órdenes mejor representados son Chiroptera (1.167 ejemplares) y Rodentia (725 ejemplares) con 123 y 57 especies respectivamente (Tabla 1). Los especímenes proceden de los departamentos de Amazonas (80 especímenes), Antioquia (1), Boyacá (15), Cundinamarca (705), Caquetá (58), Casanare (25), Cesar (1), Chocó (106), Córdoba (13), Guaviare (1), Huila (198), Meta (354), Nariño (5), Putumayo (186), Santander (43), Tolima (112), Valle del Cauca (2) y Vichada (16).
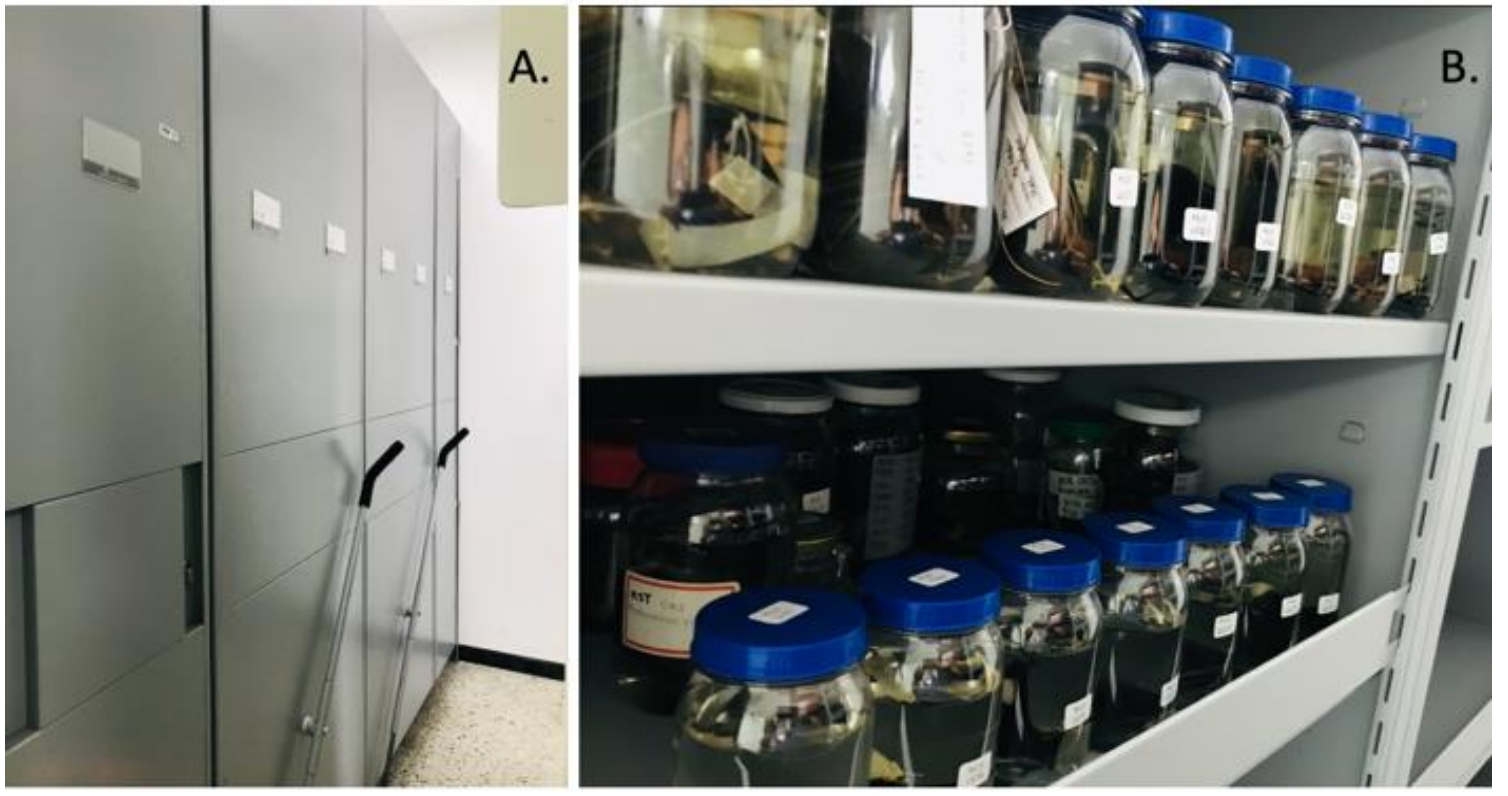

FIGURA 1. A. Infraestructura de almacenamiento de especímenes. B. Colección en líquido Museo de Historia Natural de la Universidad Distrital Francisco José de Caldas (MHNUD-M).
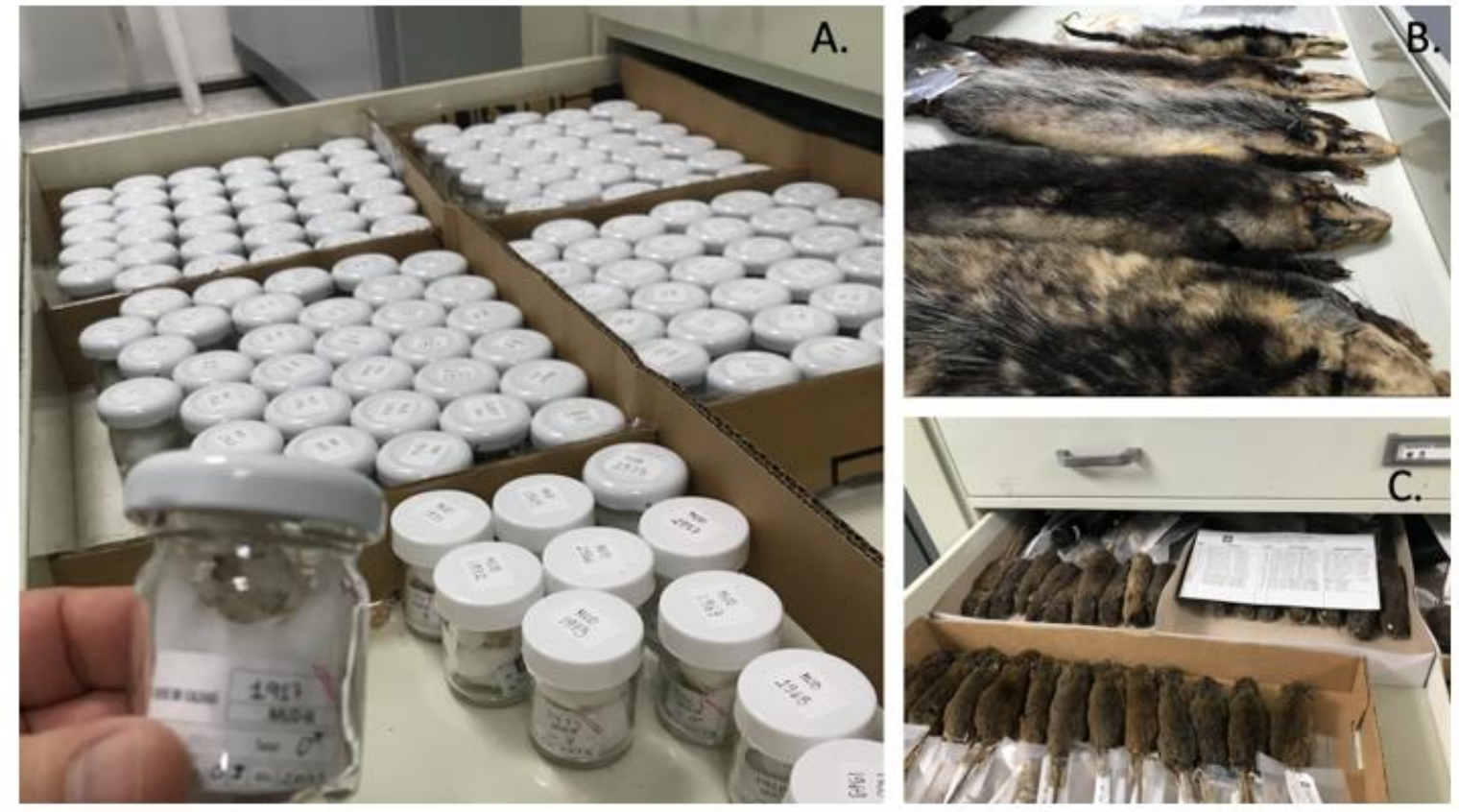

FIGURA 2. A. Colección de cráneos extraídos de Mamíferos en líquido. B. Colección de pieles en seco. C. Colección en seco de pieles, cráneos y esqueletos. Museo de Historia Natural de la Universidad Distrital Francisco José de Caldas (MHNUD-M). 
Especímenes de la colección de mamíferos del MHNUD-M han sido citados en al menos 19 publicaciones científicas (Alarcón-Bernal et al. 2006; Pardo-Martínez et al. 2006; GonzálezMaya et al. 2011; Pinto et al. 2012; Ramírez-Chaves et al. 2012, 2020; Pinilla-Cortes et al. 2013; Rodríguez-Bolaños et al. 2013, 2015a, 2015b, 2017; Castellanos-Flórez \& Rodríguez-Bolaños 2016; Esquivel et al. 2016, 2017, 2020; Aya-Cuero et al. 2017, 2019; Bautista et al. 2018; Noguera-Urbano et al. 2019; Torres-Martínez et al. 2019).

Registros de los órdenes Artiodactyla, Chiroptera, Cingulata, Rodentia y Primates procedentes de cinco departamentos pueden ser consultados en línea (Rodríguez-Bolaños et al. 2017). Este recurso se encuentra disponible en: https://ipt.biodiversidad.co/sib/resource?r=mamif_ud

TABLA 1. Órdenes, familias, géneros y especies de mamíferos depositados en la colección de Museo de Historia Natural de la Universidad Distrital Francisco José de Caldas (MHNUD-M).

\begin{tabular}{|c|c|c|c|c|}
\hline Orden & Familias & Géneros & Especies & $\begin{array}{l}\text { Número de } \\
\text { especímenes }\end{array}$ \\
\hline Didelphimorphia & 1 & 13 & 16 & 48 \\
\hline Paucituberculata & 1 & 1 & 1 & 13 \\
\hline Cingulata & 1 & 2 & 4 & 6 \\
\hline Pilosa & 3 & 3 & 3 & 3 \\
\hline Sirenia & - & - & - & - \\
\hline Eulipotyphla & 1 & 1 & 3 & 28 \\
\hline Chiroptera & 7 & 46 & 123 & 1167 \\
\hline Carnivora & 4 & 8 & 8 & 29 \\
\hline Perissodactyla & - & - & - & - \\
\hline Artiodactyla & 1 & 1 & 1 & 3 \\
\hline Cetacea & - & - & - & - \\
\hline Primates & 4 & 5 & 6 & 6 \\
\hline Rodentia & 10 & 36 & 57 & 723 \\
\hline Lagomorpha & 1 & 1 & 1 & 4 \\
\hline Total & 37 & 116 & 223 & 2030 \\
\hline
\end{tabular}

Muchos estudiantes del grupo de investigación Biodiversidad de Alta Montaña (BAM) y profesionales externos han contribuido al enriquecimiento del MHNUD-M, ya sea mediante la realización de sus trabajos de grado (Maritza González, Sandra Ramírez, Sandra Milena Alarcón, Jenny Gallo, Dayanne Carvajal, Paola Lemus, Pilar Galeano, Johan Gantiva, Catalina Pinilla, Sebastián Bautista, Ana María Guerrero, Andrés Otálora, Evelyn Rodríguez, Ivonne Rodríguez, Beatriz Rodríguez, Cesar Meza, Carlos Aya, Diana Camelo, Diana Piña, Faryd Pinilla, Catalina Torres Palacios, Alejandra García, Diego Alejandro Forero, José Moreno, Diego Esquivel, Deiris Romero, Angie Solórzano, Lizeth Corredor, Luis Arcos, Camila Díaz), o como profesionales vinculados a convenios de cooperación científica suscritos con entidades como la Corporación para el Desarrollo Sostenible del Sur de la Amazonía (CORPOAMAZONIA), Corporación Autónoma del Alto Magdalena (CAM), Corporación para el Desarrollo Sostenible de la Macarena (CORMACARENA), Instituto de Investigaciones de Recursos Biológicos Alexander von Humboldt (IAvH), Secretaria de Ambiente de Bogotá y algunas entidades privadas. Otras localidades se han explorado mediante la gestión de expediciones de interés particular del curador de la colección, o durante las salidas de campo de asignaturas como Mastozoología, Diversidad y Conservación de Mamíferos Neotropicales y Zoología de Vertebrados.

La colección científica MHNUD-M es un escenario de formación para los nuevos mastozoólogos del BAM que se vinculan con funciones de depuración y organización, o 
como investigadores en formación en proyectos específicos. Entre los estudiantes que aportan a la organización actual se encuentran: Yesenia Salinas, Nicoll Castiblanco, Michael Guerrero, Esteban Acosta, Vivian Agudelo, Jhony Cruz y la profesional Lucía Rodríguez quien ha cumplido un papel clave en la depuración y tratamiento de especimenes al ingresar a la colección.

Un aporte importante hacia el conocimiento de los roedores depositados en MHNUD-M ha estado liderado por Ronald Castellanos, investigador del BAM, quien ha dedicado más de seis años al estudio de este grupo y actualmente participa en proyectos editoriales relacionados con productos derivados de ese trabajo.

\section{AGRADECIMIENTOS}

Al Grupo de Investigación Biodiversidad de Alta Montaña (BAM), a las entidades con las que hemos suscrito convenios o acuerdos de cooperación y a un sinnúmero de comunidades rurales que nos han acogido durante estos últimos 20 años.

\section{REFERENCIAS}

Alarcón-Bernal S, Hernández-Jaramillo L, Rodríguez-Bolaños A. 2006. Estado del Arte de la Colección de Mamíferos del Museo de Historia Natural de la Universidad Distrital Francisco José de Caldas. In: Andrade-C MG, Aguirre J, Rodríguez-Mahecha JV, editors. Segundo Congreso Colombiano de Zoología. Libro de Resúmenes. Editorial Panamericana Formas e Impresos. S.A. Pp. 383-384.

Aya-Cuero C, Rodríguez-Bolaños, Superina M. 2017. Population density, activity patterns, and ecological importance of giant armadillos (Priodontes maximus) in Colombia: Journal of Mammalogy 98:770-778. https://doi.org/10.1093/jmammal/gyx006

Aya-Cuervo C, Mosquera-Guerra F, Esquivel DA, Trujillo F, Brooks D. 2019. Medium and large mammals of the mid Planas River basin, Colombia. Biota Colombiana 20:76-92.

http:/ / dx.doi.org/10.21068/c2019.v20n02a06

Bautista Plazas S, Rodríguez-Bolaños A, Castellanos-Flórez R. 2018. First record of eastern cloud forest rat Nephelomys nimbosus (Rodentia: Cricetidae) for Colombia. Therya 9:103-106.

http:/ / dx.doi.org/10.12933/therya-18-431

Castellanos-Flórez R, Rodríguez-Bolaños A. 2016. Roedores. Colección de Mastozoología. Museo de Historia Natural, Universidad Distrital Francisco José de Caldas. In: Villareal D, Becerra F, Fonseca G, Quintero G, editors. El impacto de la investigación en ciencias biológicas y su enseñanza. Bogotá: Editorial UD.

Esquivel DA, Penagos AP, García RS, Bennett D. 2020. New records of Pygmy Round-eared Bat, Lophostoma brasiliense Peters, 1867 (Chiroptera, Phyllostomidae), and updated distribution in Colombia. Check List 16:277-285. https: / / doi.org/10.15560/16.2.277

Esquivel DA, Camelo-Pinzón D, Rodríguez-Bolaños A. 2017. New record of bilateral hyperdontia in Carollia brevicauda (Chiroptera: Phyllostomidae). Therya 8:71-73. http://dx.doi.org/10.12933/therya-17-440

Esquivel DA, Camelo-Pinzón D, Rodríguez-Bolaños A. 2016. Bats of the Guayupes Forest, Acacias, Meta, Colombia. Fieldguides.fieldmuseum.org (771) Versión 1 06/2016.

González-Maya J, Rodríguez-Bolaños A, Pinto D, Jiménez-Ortega MA. 2011. Recent confirmed records and distribution of the White-nosed Coati Nasua narica in Colombia. Small Carnivore Conservation 45:26-30. 
Noguera-Urbano EA, Colmenares-Pinzón JE, Villota J, Rodríguez-Bolaños A, Ramírez-Chaves HE. 2019. Shrews (Cryptotis) of Colombia: What do we know about them? Therya 10(2):131-147. https:// doi.org/10.12933/therya-19-760

Pinilla-Cortes C, Rodríguez-Bolaños A, Vogtschmidt S. 2013. Descripción de los pulsos de ecolocación de Phyllostomus hastatus Pallas 1767, en un bosque húmedo tropical de San Francisco, Cundinamarca - Colombia. Revista Biodiversidad Neotropical 2:106-112. https:// doi.org/10.18636/ bioneotropical.v3i2.164

Pinto D, Rodríguez-Bolaños A, Jiménez-Ortega MA. 2012. Mamíferos del Valle (Bahía Solano-Chocó). Una aproximación al conocimiento de la dinámica de la relación Fauna y Comunidad Local. Editorial Académica Española.

Ramírez-Chaves HE, Carvajal-Agudelo JD, Hoyos MR. Bustamante-Manrique S, Castaño-Rivera A, Rivillas-Carmona MA, Ossa-López PA, Rivera-Páez FA 2020. New records and confirmation of the presence of three species of primates (Mammalia, Primates) in southwestern Colombia. Check List 16 (4):811-819. https:/ / doi.org/10.15560/16.4.811

Ramírez-Chaves HE, Mulder KP, Marín D, Pérez WA, Martínez-Arias VM. 2012. Has Colombian Weasel Mustela felipei been overlooked in collections? Small Carnivore Conservation 47:30-34.

Rodríguez- Bolaños, A., Solórzano, A., 2017. Colección Mastozoológica de la Universidad Distrital Francisco José de Caldas. v1.0. Universidad Distrital Francisco José de Caldas. Dataset/Occurrence. http:// doi.org/10.15472/dcmcu7

Rodríguez-Bolaños A, Carvajal L, Ariza W. 2015. Fauna de los bosques de la cuenca de los ríos Planas y Tillavá, Puerto Gaitán, Meta, Colombia. Editorial Corporación para el Desarrollo Sostenible del Área de Manejo Especial La Macarena.

Rodríguez-Bolaños A, Carvajal L, Ariza W. 2015. Fauna del Bosque de los Guayupes. Cuenca del Río Guayuriba - Acacias Meta. Editorial Corporación para el Desarrollo Sostenible del Área de Manejo Especial La Macarena.

Rodríguez-Bolaños A, Gonzales-Caro S, Etter A, Stevenson P. 2013. Predictive models of the geographic distribution of Ateline monkeys (Lagothrix y Ateles) in Colombia, In: Defler, T.R., Stevenson, P.R., Bueno, M. L., Guzman-Caro, D, C., editors. Primates Colombianos en Peligro de Extinción, Asociación Primatológica Colombiana. 194-216 pp.

Pardo-Martínez RA, Rodríguez-Bolaños A. 2006. Caracterización de pequeños mamíferos en la vereda de Noruega Alta, Municipio de Silvana, In: Andrade-C MG, J. Aguirre, J. V. RodríguezMahecha, editors. Segundo Congreso Colombiano de Zoología. Libro de Resúmenes. Editorial Panamericana Formas e Impresos. S.A.

Torres-Martínez MM, Ramírez-Chaves HE, Noguera-Urbano EA, Colmenares-Pinzón JE, Passos FC, García J. 2019. On the distribution of the Brazilian porcupine Coendou prehensilis (Erethizontidae) in Colombia. Mammalia 83:290-297. https://doi.org/10.1515/mammalia-20180043

Editor: Miguel E. Rodríguez-Posada

Recibido 2020-05-07

Revisado 2020-05-17

Aceptado 2020-05-25

Publicado 2020-07-05 